\title{
proEQUIB: IDL Library for Plasma Diagnostics and Abundance Analysis
}

\author{
Ashkbiz Danehkar ${ }^{1,2}$
}

1 Research Centre in Astronomy, Astrophysics and Astrophotonics, Macquarie University, Sydney, NSW 2109, Australia 2 Harvard-Smithsonian Center for Astrophysics, 60 Garden Street, Cambridge, MA 02138, USA

DOI: $10.21105 /$ joss.00899

\section{Software \\ - Review ca \\ - Repository c \\ - Archive c}

Submitted: 30 June 2018

Published: 04 December 2018

\section{License}

Authors of papers retain copyright and release the work under a Creative Commons Attribution 4.0 International License (CC-BY).

\section{Summary}

The emission lines emitted from gaseous nebulae carry valuable information about the physical conditions and chemical abundances of ionized gases in these objects, as well as the interstellar reddening. We determine the electron temperature, the electron density, and the ionic abundances from the dereddened fluxes of collisionally excited lines (CEL) and recombination lines (RL) identified in nebular spectra (see e.g. Danehkar, Parker, \& Ercolano (2013); Danehkar, Todt, Ercolano, \& Kniazev (2014); Danehkar, Parker, \& Steffen (2016); Danehkar (2018a)).

proEQUIB is a library including several application programming interface (API) functions developed in the Interactive Data Language (IDL), which can be used to determine temperatures, densities, and chemical abundances from emission lines of ionized nebulae. This IDL library can also be used by the GNU Data Language (GDL) (Arabas, Schellens, Coulais, Gales, \& Messmer (2010); Coulais et al. (2010)), which is a free and open-source alternative IDL compiler. This IDL/GDL package employs the IDL library AtomNeb Atomic Data for Ionized Nebulae (Danehkar (2018b)), which contains collision strengths and transition probabilities for collisional excitation calculations, and recombination coefficients for recombination calculations. This package includes several API functions to determine physical conditions and chemical abundances from CEL and RL, derive interstellar extinctions from Balmer lines, and deredden the observed fluxes:

- The API functions for the CEL analysis were developed in the IDL programming language based on the algorithm of the FORTRAN program EQUIB (Howarth \& Adams (1981); Howarth et al. (2016)), which calculates atomic level populations and line emissivities in statistical equilibrium in multi-level atoms for the given physical conditions. These API functions can be used to determine the electron temperature, the electron density, and the ionic abundances from the dereddened fluxes of collisionally excited lines emitted from ionized gaseous nebulae.

- The API functions for the $R L$ analysis were developed in IDL according to the algorithm of the recombination scripts by X. W. Liu and Y. Zhang included in the FORTRAN program MOCASSIN (Ercolano, Barlow, Storey, \& Liu (2003); Ercolano, Barlow, \& Storey (2005)). These API functions can be used to determine the ionic abundances from the dereddened fluxes of recombination lines emitted from ionized nebulae.

- The API functions for the reddening analysis were developed based on the methods of the reddening functions from the Space Telescope Science Data Analysis System 
(STSDAS) IRAF Package (Bushouse \& Simon (1994); Shaw \& Dufour (1994)). These API functions can be employed to obtain interstellar extinctions for different reddening laws from the observed fluxes of Balmer lines detected in nebular spectra, and deredden the measured fluxes of emission lines.

proEQUIB has recently been used for plasma diagnostics and abundance analysis of some planetary nebulae (Danehkar et al. (2016); Danehkar (2018a)). This IDL/GDL package heavily relies on the IDL Astronomy User's library (Landsman (1993); Landsman (1995)) and the IDL library AtomNeb (Danehkar (2018b)). The API functions of this IDL library can easily be utilized to generate spatially-resolved maps of extinction, temperature, density, and chemical abundances from integral field spectroscopic observations (see e.g. Danehkar et al. (2013); Danehkar et al. (2014); Danehkar (2014)).

\section{Acknowledgements}

A.D. acknowledges the receipt of a Macquarie University Research Excellence Scholarship.

\section{References}

Arabas, S., Schellens, M., Coulais, A., Gales, J., \& Messmer, P. (2010). GNU Data Language (GDL) - a free and open-source implementation of IDL. In EGU general assembly conference, Geophysical research abstracts (Vol. 12, p. 924).

Bushouse, H., \& Simon, B. (1994). The IRAF/STSDAS Synthetic Photometry Package. In D. R. Crabtree, R. J. Hanisch, \& J. Barnes (Eds.), Astronomical data analysis software and systems iii, Astronomical society of the pacific conference series (Vol. 61, p. 339).

Coulais, A., Schellens, M., Gales, J., Arabas, S., Boquien, M., Chanial, P., Messmer, P., et al. (2010). Status of GDL - GNU Data Language. In Y. Mizumoto, K.-I. Morita, \& M. Ohishi (Eds.), Astronomical data analysis software and systems xix, Astronomical society of the pacific conference series (Vol. 434, p. 187).

Danehkar, A. (2014). Evolution of Planetary Nebulae with WR-type Central Stars (PhD thesis). Macquarie University, Australia.

Danehkar, A. (2018a). Bi-Abundance Ionisation Structure of the Wolf-Rayet Planetary Nebula PB 8. Publications of the Astronomical Society of Australia, 35, e005. doi:10.1017/pasa.2018.1

Danehkar, A. (2018b). AtomNeb: Atomic Data for Ionized Nebulae. The Journal of Open Source Software, submitted.

Danehkar, A., Parker, Q. A., \& Ercolano, B. (2013). Observations and three-dimensional ionization structure of the planetary nebula SuWt 2. Monthly Notices of the Royal Astronomical Society, 434, 1513-1530. doi:10.1093/mnras/stt1116

Danehkar, A., Parker, Q. A., \& Steffen, W. (2016). Fast, Low-ionization Emission Regions of the Planetary Nebula M2-42. The Astronomical Journal, 151, 38. doi:10.3847/0004$6256 / 151 / 2 / 38$

Danehkar, A., Todt, H., Ercolano, B., \& Kniazev, A. Y. (2014). Observations and three-dimensional photoionization modelling of the Wolf-Rayet planetary nebula Abell 48. Monthly Notices of the Royal Astronomical Society, 439, 3605-3615. doi:10.1093/mnras/stu203 
Ercolano, B., Barlow, M. J., \& Storey, P. J. (2005). The dusty MOCASSIN: fully selfconsistent 3D photoionization and dust radiative transfer models. Monthly Notices of the Royal Astronomical Society, 362, 1038-1046. doi:10.1111/j.1365-2966.2005.09381.x

Ercolano, B., Barlow, M. J., Storey, P. J., \& Liu, X.-W. (2003). MOCASSIN: a fully three-dimensional Monte Carlo photoionization code. Monthly Notices of the Royal Astronomical Society, 340, 1136-1152. doi:10.1046/j.1365-8711.2003.06371.x

Howarth, I. D., \& Adams, S. (1981). Program EQUIB. University College London.

Howarth, I. D., Adams, S., Clegg, R. E. S., Ruffle, D. P., Liu, X.-W., Pritchet, C. J., \& Ercolano, B. (2016). EQUIB: Atomic level populations and line emissivities calculator. Astrophysics Source Code Library, ascl:1603.005.

Landsman, W. B. (1993). The IDL Astronomy User's Library. In R. J. Hanisch, R. J. V. Brissenden, \& J. Barnes (Eds.), Astronomical data analysis software and systems ii, Astronomical society of the pacific conference series (Vol. 52, p. 246).

Landsman, W. B. (1995). The IDL Astronomy User's Library. In R. A. Shaw, H. E. Payne, \& J. J. E. Hayes (Eds.), Astronomical data analysis software and systems iv, Astronomical society of the pacific conference series (Vol. 77, p. 437).

Shaw, R. A., \& Dufour, R. J. (1994). The FIVEL Nebular Modelling Package in STSDAS. In D. R. Crabtree, R. J. Hanisch, \& J. Barnes (Eds.), Astronomical data analysis software and systems iii, Astronomical society of the pacific conference series (Vol. 61, p. 327). 